\title{
Optimum Design for Spring with Orthogonal Design Method
}

\author{
Han Fu-Jie \\ Electromagnetic Engineering College Henan Agricultural University \\ Zhengzhou City, Henan Province. P. R. China
}

\begin{abstract}
:
Orthogonal design method is a sort of direct optimum method, by which the optimal result may be obtained through simple computation and analysis. This method has gained increasingly extensive interest in scientific research, engineering design, and production management as well.

The orthogonal table is the basic instrument for carrying out optimum design with orthogonal design method. It is a mathematical table established on the basis of the idea of isostatic distribution. As various levels may appear in any column, it makes a partial experiment contain all levels of all factors; furthermore, all combinations of any two columns may appear, which makes any experiment between two factors become overall experiment. Though the arrangement of the orthogonal table is only for a partial experiment, nevertheless it may lead to understand the conditions of overall experiment.

While making optimum design with orthogonal table, it may use the design variables of the mathmatical model for optimum design for the factors of orthogonal table, and use the objective function $f(X)$ for the experiment target. By means of orthogonal table for the arrangement of design plan, it is easy to determine an optimum design plan.

This article recommends the basic principles of using orthogonal design method to proceed with optimal design, and the process of how to use orthogonal design method for the optimization of spring.

From the theory and examples of this article, it may clearly understand the superiority of applying orthogonal design method to conduct optimum design. The optimum design can be obtained through simple computation. It is particularly suitable to conditions that the design variables are integer, or discrete variables.
\end{abstract}

\section{0 Introduction}

Orthogonal design method is a sort of direct optimum method, by which the optimum result may be obtained through simple computation and analysis. This is why the application of orthogonal design method has gained increasingly extensive interest in scientific research, engineering design, and production management as well.

\section{0 The Basic Principles of Orthogonal Design Method For Optimum Design :}

The orthogonal table is the basic instrument for carrying out optimum design with orthogonal design method. It is a mathematical table established on the basis of the idea of isostatic distribution. The general orthogonal table with equal levels can be written as $L_{\mathbf{a}}\left(b^{c}\right)$, in which " $\mathrm{L}$ " indicates orthogonal table, " $\mathrm{a}$ " indicates the rows of orthogonal table, that is the number of experiments to be taken when experiments are arranged by orthogonal table; " $c$ " indicates the columns of orthogonal table, that is the number of factors that the orthogonal 
table can arrange at most; " $b$ " indicates the number of levels of orthogonal table in the arrangement of factors, that is the different values obtained by factors in experimental designs.

Let's take a most simple orthogonal table $\mathrm{L}_{4}\left(2^{3}\right)$ (see Table 1 ) for observation. We can see :

Table $1 \quad \mathrm{~L}_{4}\left(2^{3}\right)$

\begin{tabular}{|c|c|c|c|}
\hline Column No. & 1 & 2 & 3 \\
\hline 1 & 1 & 1 & 1 \\
\hline 2 & 1 & 2 & 2 \\
\hline 3 & 2 & 1 & 2 \\
\hline 4 & 2 & 2 & 1 \\
\hline
\end{tabular}

(1) Each level appears in any columns with equal times of appearance.

(2) All combinations of various levels appear in any two columns, and the joint arrangement is isostatic.

As various levels may appear in any column, it makes a partial experiment contain all levels of all factors; furthermore, all combinations of any two columns may appear, which makes any experiment between two factors become overall experiment. Though the arrangement of the orthogonal table is only for a partial experiment, nevertheless it may lead to understand the conditions of overall experiment.

While making optimum design with orthogonal table, it may use the design variables of the mathematical model for optimum design for the factors of orthogonal table, and use the objective function $f(X)$ for the experiment target. By means of orthogonal table for the arrangement of design plan, it is easy to determine an optimum design plan.

\section{0 A practical example is shown below to introduce how to make optimum design for spring} by using the method of orthogonal design.

Example: Try to design a cylinder spring with compressed spirals. Its deformed dimen$\operatorname{sion} \lambda=16.59 \mathrm{~mm}$. The maximum working temperature is $150^{\circ} \mathrm{C}$, material requires chromium vanadium steel 50CrVA. The desired working life is $10^{5}$ cycle index. After intensified treatment, its allowable shearing stress $[\tau]=404.9 \mathrm{MPa}$. The required rigidity of spring $\mathrm{c}=41 \mathrm{~N} /$ mm. The axial pressure that the spring endures $F=680.2 \mathrm{~N}$. Try to determine the wire diameter $d$, and medium diameter of the spring $D$, and number of effective coils $n$. It is requested to design, under the conditions to meet the strength and rigidity requirements, a structure plan in minimum weignt:

Solution: 3.1 Write out the optimum mathematical model of this example.

3. 1. 1. Design variables

Design variables are the wire diameter $d$, medium diameter of the spring $D$, and number of coils $n$.

$\therefore \mathrm{X}=\left[\mathrm{x}_{1}, \mathrm{x}_{2}, \mathrm{x}_{3}\right]^{\mathrm{T}}=[\mathrm{d}, \mathrm{D}, \mathrm{n}]^{\mathrm{T}}$

3. 1.2. Objective function

Taking the number of noneffective coils $N_{1}=1.8$. The weight target of the spring to be calculated by its volume is :

$$
\begin{aligned}
& V=\frac{\pi_{2}}{4} d^{2} D n+\frac{\pi_{2}}{4} d^{2} D n_{1} \\
& \therefore f(X)=\frac{\pi^{2}}{4} d^{2} D n+\frac{\pi^{2}}{4} d^{2} D n_{1}=2.47 x_{1}^{2} x_{2} x_{3}+4.44 x_{1}^{2} x_{2}
\end{aligned}
$$




\section{1. 3. Design Constraints}

Basing on the strength requirement of the spring,

$$
\tau=\frac{8 k_{1} F D}{\pi d^{3}} \leqslant[\tau]
$$

in which $\mathbf{k}_{1}-$ curvature coefficient.

$$
\mathbf{k}_{1}=\frac{4 \mathrm{C}-1}{4 \mathrm{C}-4}+\frac{0.615}{\mathrm{C}}=\frac{1.6}{\left(\frac{\mathrm{D}}{\mathrm{d}}\right)^{0.14}}
$$

$\mathrm{C}-$ spring exponent, $\mathrm{C}=\frac{\mathrm{D}}{\mathrm{d}}$

$\mathrm{F}$ - Axial pressure endured by the spring, N.

From this computation we can have:

$$
\begin{aligned}
g_{1}(X) & =\frac{8 k_{1} F D}{\pi d^{3}[\tau]}-1=8 \cdot \frac{1.6}{\left(\frac{D}{d}\right)^{0.14}} \cdot \frac{F D}{\pi d^{3}[\tau]}-1 \\
& =6.84 \mathrm{x}_{1}^{-2.86} \mathrm{x}_{2}^{0.86}-1 \leqslant 0
\end{aligned}
$$

Basing on the figidity requirement of the spring

$$
\mathrm{n}=\frac{\mathrm{Gd}^{4}}{8 \mathrm{cD} \mathrm{D}^{3}} \text {. }
$$

where, G-shearing elastic modulus of the spring material, and its value here is $8 \times 10^{4} \mathrm{MPa}$ From this we can have

$$
\mathrm{g}_{2}(\mathrm{X})=\frac{\mathrm{Gd}^{4}}{8 \mathrm{cD}^{3} \mathrm{n}}-1=243.90 \mathrm{x}_{1}^{4} \mathrm{x}_{2}^{-3} \mathrm{x}_{3}^{-1}-1 \leqslant 0
$$

According to experience,

$$
4 \leqslant \frac{\mathrm{D}}{\mathrm{d}} \leqslant 14
$$

From this, we can have

$$
\begin{aligned}
& g_{3}(X)=4-\frac{x_{2}}{x_{1}} \leqslant 0 \\
& g_{4}(X)=\frac{x_{2}}{x_{1}}-14 \leqslant 0
\end{aligned}
$$

From the analysis shown above, it is known that the optimum mathematical model of this example is

$$
\begin{array}{ll}
\min f(X) & =2.47 x_{1}^{2} x_{2} x_{3}+4.44 x_{1}^{2} x^{2} \\
\text { s. t. } & g_{1}(X)=6.84 x_{1}^{-2.86} x_{2}^{0.86}-1 \leqslant 0 \\
& g_{2}(X)=243.90 x_{1}^{4} x_{2}^{-3} x_{3}^{-1}-1 \leqslant 0 \\
& g_{3}(X)=4-\frac{x_{2}}{x_{1}} \leqslant 0 \\
& g_{4}(X)=\frac{x_{2}}{x_{1}}-14 \leqslant 0 \\
x_{1}, x_{2}, x_{3}>0
\end{array}
$$

3. 2 Select an appropriate orthogonal table for the preparation of orthogonal design plan.

As the design variables, i. e. factors have been determined, the number of factors' levels can be determined by experience basing on the intention of the subject. If no experience in this area, the original value may be given first, and then try to find another level values according to geometric series or arithmatic series. The selected orthogonal table should be able to contain all factors needed. A sheet of orthogonal table is only use for one round of design, or it may have several sheets of orthogonal tables combined into one, if necessary. When a round of orthogonal tables has completed the selection of optimum goal, it is also necessary to decide whether another round is necessary to be carried out according to the result of this round.

This example is composed of three design variables, and three numerical values are taken from each design variable. Thus a table of factor levels is formed. (see table 2)

The selected orthogonal table should be able to contain all factors and all factor levels. It 
can be seen from this point that it is appropriate to select orthogonal table $L_{9}\left(3^{4}\right)$ (See table 3)

Table 2 Table of factor levels

\begin{tabular}{|c|c|c|c|}
\hline level factor & d & D & n \\
\hline 1 & 3 & 12 & 5 \\
\hline 2 & 5 & 20 & 10 \\
\hline 3 & 10 & 30 & 20 \\
\hline
\end{tabular}

Table 3

\begin{tabular}{|c|c|c|c|c|c|}
\hline \multirow{2}{*}{ Factors } & \multicolumn{3}{|c|}{ Design variables } & \multirow{2}{*}{$\begin{array}{c}\text { pbjective functions } \\
\mathrm{f}(\mathrm{X})\end{array}$} & \multirow{2}{*}{$\begin{array}{l}\text { Constraints } \\
g(X)\end{array}$} \\
\hline & $x_{1}(d)$ & $\mathrm{x}_{2}(\mathrm{D})$ & $\mathrm{x}_{3}(\mathrm{n})$ & & \\
\hline 1 & (1) 3 & (1) 12 & (1) 5 & 1813.32 & Constraints violated \\
\hline 2 & (1) 3 & (2) 20 & (2)10 & 5245.2 & Contraints violated \\
\hline 3 & (1) 3 & (3) 30 & (3) 20 & 14536.8 & Constraints violated \\
\hline 4 & (2) 5 & (1) 12 & (2) 10 & 8742 & Constraints violated \\
\hline 5 & (2) 5 & (2) 20 & (3) 20 & 26920 & Constraints Satisfied \\
\hline 6 & (2) 5 & (3) 30 & (1) 5 & 12592.5 & Constraints violated \\
\hline 7 & (3)10 & (1) 12 & (3) 20 & 64608 & Constraints violated \\
\hline 8 & (3) 10 & (2) 20 & (1) 5 & 33580 & Constraints violated \\
\hline 9 & (3)10 & (3) 30 & (2) 10 & 87420 & Constraints violated \\
\hline$Y_{\mathrm{i} 1}$ & 21595.32 & 75163.32 & 47985.82 & & \\
\hline $\mathrm{Y}_{\mathrm{j} 2}$ & 48254.5 & 65745.2 & 101407.2 & & \\
\hline $\mathrm{Y}_{\mathrm{j} 3}$ & 185608 & 114549.3 & 106064.8 & & \\
\hline$R_{j}$ & 164013 & 48804.1 & 53421.38 & & \\
\hline good level & $\mathrm{d}_{1}$ & $\mathrm{D}_{2}$ & $\mathrm{n}_{1}$ & & \\
\hline $\begin{array}{c}\text { Primary and } \\
\text { secondary factors }\end{array}$ & \multicolumn{3}{|c|}{$\mathrm{d}, \mathrm{n}, \mathrm{D}$} & & \\
\hline $\begin{array}{l}\text { Optimum } \\
\text { combination }\end{array}$ & \multicolumn{3}{|c|}{$d_{1} D_{2} n_{1}$} & & \\
\hline
\end{tabular}

A factor can be arranged in each column of the orthogonal table, and d, D. n are arranged respectively in the first three columns of $L_{9}\left(3^{4}\right)$, and no factor can be put in column 4 , which has no effect in orthogonal design, thereby it may be eliminated from the table. Then the different figures of all columns in the orthogonal table can be changed into corresponding levels of the homologous factors.

Combine it to this example, the first column is occupied by $\mathrm{d}$, and $3(\mathrm{~mm})$ are written behind the three numerals " 1 " of the first column, that is level 1 corresponds to factor $d$; and 5 $(\mathrm{mm})$-that is level 2 of $\mathrm{d}$, are written behind the three numerals " 2 " of the first column: and $10(\mathrm{~mm})$ that is the level 3 of $\mathrm{d}$, are written behind the three numerals " 3 " of the first column. The remaining two columns are anagolous. And in this way an orthogonal design plan is formed. Every horrizontal line of the table represents a design plan. If the value of the objec- 
tive function of every design plan can be figured out and the constraints are examined whether satisfied or not, the result shown in Table 3 is obtained.

3. 3 Analysis of the design result.

It is apparent from table 3 that the value of objective function of the 5 th design plan is in the middle, however, it may satisfy all the constraints, which is the better combination.

$X=\left[x_{1}, x_{2}, x_{3}\right]^{T}=[d, D, n]^{T}=[5,20,20]^{T}, f(X)=26920$

Whether this design plan is optimum combination or not, it can be simply (or through audio-visual) analyzed by using the method of maximum difference. In table $3, Y_{j k}$ is the sum of objective functions of design plans corresponding to $\mathrm{j}$ factor of $\mathrm{K}$ level. The good level combination of various factors is the optimum combination. The reason is the value of objective function being "the less the better". It can be seen from table 3 that the optimum combination of this example would be $d_{1} D_{2} n_{1}$. $R_{j}$ in table 3 is the maximum difference of factor $j$. Its computing formula is :

$$
R_{j}=\max \left[Y_{j 1}, Y_{j 2}, Y_{j 3}\right]-\min \left[Y_{j 1}, Y_{j 2}, Y_{j 3}\right]
$$

The bigger the $R_{j}$, means the bigger the influence of the design variable on the value of the objective functions. The primary and secondary design variables can be judged from this. It is clearly seen from table 3 that the primary and secondary sequence of factors in this example is $d, n, D$. The better combination directly obtained from the table is $d_{2} D_{2} n_{3}$. It shows that there probably exists another best design plan. Since $d$ and $n$ are all discrete variables, if a smaller value is taken (if $d=4.5 \mathrm{~mm}, \mathrm{n}=19$ ) though the values of objective functions were decreased, nevertheless they can not satisfy the constraints. Therefore the optimum solution is still like this :

$X^{*}=[d, D, n]^{T}=[5,20,20]^{\mathrm{T}}, f\left(X^{*}\right)=26920$

If this example can be determined by using the method of geometric programme, it may obtain :

$\mathrm{x}_{\mathrm{i}}^{*}=\mathrm{d}=4.89 \mathrm{~mm}$

$\mathbf{x}_{\mathbf{2}}^{*}=\mathrm{D}=20.01 \mathrm{~mm}$

$\mathbf{x}_{3}^{*}=\mathbf{n}=14.57$

According to related standards and practical conditions, $5 \mathrm{~mm}$ can be used for the diagram of the spring wire, medium diameter of the spring can be $20 \mathrm{~mm}$ round, and the number of effective coils can be 15 round. Its value of the objective function can be 20745 . If compared with this example, it is obvious that the design plan is comparatively better. If $d=5 \mathrm{~mm}, D=$ $20 \mathrm{~mm}, \mathrm{n}=15$, suppose these figures are used in the original optimum mathematical model, however, they can hardly meet the constraints. This is why the optimum solution determined from this example is still the optimum solution.

\section{0 Conclusion:}

4. 1 The application of orthogonal design method to handle optimal design problems is a direct optimization method. The overall conclusion can be obtained through a little computation.

4. 2 It can be seen from the result of this article the superiority of applying optimum design with orthogonal design method, and it is particularly suitable to conditions that the design variables are integer, or discrete variables.

\section{Reference Documents}

1. Chen L. Z.; "Engineering Optimum Design Method", Metallurgical Industry Press, 1985, PP. 181-185.

2. Zhang R. T. ; "Propability Statistic", CCBT University Press, 1987, PP. 204-220. 